\title{
A method to optimise the materials layout of small wind turbine blades
}

\author{
Mariana S.P. Costa ${ }^{*}$, Samuel P. Evans, David R. Bradney, and Philip D. Clausen \\ School of Engineering, Faculty of Engineering and Built Environment, The University of Newcastle, Newcastle, Australia
}

Received: 20 February 2017 / Received in final form: 7 July 2017 / Accepted: 27 July 2017

\begin{abstract}
This paper presents a method to optimise the material layout of a fibreglass-reinforced composite constructed small wind turbine blade. The method was developed with the intent of reducing blade mass without compromising the blade's structural performance or longevity. The bi-directional evolutionary layout optimisation method utilises finite element analysis to redesign the composite lay of the blade structure based on a strain energy criterion. Details of the procedure of this method are documented in the paper. The bi-directional evolutionary optimisation technique was applied to the existing structure of a $2.5 \mathrm{~m}$ composite blade from an Aerogenesis $5 \mathrm{~kW}$ wind turbine. The optimisation technique was able to reduce the current mass of the blade by $15 \%$ without any increase in blade deflection with strains kept below a fatigue endurance limit when subject to aerodynamic and centrifugal loading conditions at design conditions. The initial results from this study are promising and could help lower manufacturing costs of small wind turbine blades.
\end{abstract}

\section{Introduction}

One of the critical components of a wind turbine is its blades, as they are solely responsible for converting the kinetic energy of the wind into mechanical power to drive the turbine's generator. The shape of a working section of a wind turbine blade is mostly dictated by aerodynamic considerations, with the blade structural design dictated by ultimate and fatigue strength considerations, as well as manufacturing costs. The structural design of large wind turbine blades has been optimised due to significant investment in research and development from commercial interests to build cost effective and efficient machines for large-scale electricity generation. Small wind turbines, on the other hand, have not seen the same level of investment as large wind turbines, due mainly to little commercial interest. The work described in this paper is about bridging the gap between the research and development for large wind turbines and that for small wind turbines.

A small wind turbine is defined by IEC $61400-2$ as having a swept rotor area of less than $200 \mathrm{~m}^{2}$; this equates to a power output of up to $50 \mathrm{~kW}$ and a rotor diameter of less than $16 \mathrm{~m}$ [1]. The blades of small wind turbines have been constructed from a broad range of materials including timber, pultruded composite materials, glass-fibre and carbon-fibre reinforced composites [2]. Designing the blade structure using the simple loading method from [1] leads to

\footnotetext{
* e-mail: c3106982@uon.edu.au
}

an overly conservative design resulting in heavy and possible costly blades. Optimising the structure of a glassfibre reinforced blade not only reduces its mass but is likely to reduce manufacturing costs leading to an overall reduction in turbine cost.

Small wind turbines are mainly free yaw machines utilising a tailfin to point the turbine into the direction of the wind. Given most small wind turbines are located close to their load, these machines are likely to operate in turbulent flows which has been shown to increase loading on the blades due to high gyroscopic loading during yaw events [3]. Reducing the mass of these blades not only saves material costs but also reduces loading on other components of the wind turbine. Another important aspect of blade mass reduction is the reduction in blade inertia; low inertia blades accelerate more rapidly during start up and the turbine will respond quicker during yaw events, both leading to increased power production [4].

The optimisation method presented in this paper was adapted from the bi-directional evolutionary structural optimisation (BESO) method developed by [5]. BESO is a well-known method mostly used for topology optimisation of stationary structures [6]. Usually combined with finite element analysis (FEA), the basic idea behind the BESO method is to remove inefficient material and add material to weaker parts of the structure. BESO based algorithms work iteratively, adding or removing material to and from the design domain according to parameters such as the removal ratio of volume [7], until the prescribed volume for the structure is reached. As the method name implies, 
BESO algorithms causes the geometry of the structure to evolve towards its optimal design, within the design domain.

For the optimisation method presented in this paper, the geometry of the structure in question is not altered. This method was developed for wind turbine blades and it does not attempt to modify the blade geometry as this would alter its aerodynamic characteristics. As the method optimises the layout and type of the composite materials within the blade structure, to differ it from BESO, this new method will be called bi-directional evolutionary layout optimisation (BELO). BELO uses material properties from the existing wind turbine blade and instead of adding or removing material to and from the structure, the algorithm rearranges the type and lay of the composite location within the structure. BELO is also an iterative method and its algorithm will continue to rearrange the materials layout within the structure of the blade until no significant change in the overall strain energy of the blade is observed.

\section{Method}

BELO is an optimisation method that relies on the computational aid of two software packages; Strand7 finite element software and Matlab programming software. Furthermore, BELO relies on the use of Strand7 Application Programming Interface (API), which allows Matlab scripts to communicate with Strand7. Strand7 is used during the execution of the optimisation algorithm using the API, as well as for the analysis of the optimum blade models. Matlab is a programming software and the optimisation algorithm was implemented using API functions in a Matlab script. It is worth noting that BELO algorithms can be implemented using any combination of FE software and computational software.

During the execution of the optimisation routine, the geometry of the blade remains unchanged throughout the optimisation process. The material properties of the plate elements are changed according to the resulting strain energy on the element. Therefore, the plate elements material properties are the only characteristics in the model to change during the process.

\subsection{Requirements}

Similar to the BESO method, which requires a pre-defined design domain [5], BELO method requires an accurate finite element model of the wind turbine blade. The blade model must accurately reflect the physical blade geometrical characteristics and material properties. The model must also be correctly restrained and loaded to assure relevant and accurate results. The blade model used in the numerical example of this study was extensively tested and calibrated to match the Aerogenesis $5 \mathrm{~kW}$ wind turbine blade characteristics. Three different stationary point loads were experimentally tested and the results were used to calibrate the blade model. The load applied to the blade model for implementation of the optimisation algorithm, was calculated with a combination of blade element momentum (BEM) method and panel method, using code written by one of the authors [8]. The numerical values of the load used in the optimisation routine are only valid under the assumption of steady state conditions.

The method was developed to allow composite material properties to be assigned to every plate finite element that represents the structure of the blade. Similar to BESO algorithms used to optimise composite-constructed structures, BELO uses the strain energy of individual elements and the overall strain energy of the structure as optimisation parameters [9]. Strain energy is a quantity that relates stiffness of materials to displacement. Furthermore, FEA of composite-constructed structures is based on the energy formulation of the laminate theory [10].

\subsection{Setting up}

As the BELO method uses existing composite material properties of wind turbine blades, it is necessary to rank these properties before implementing the algorithm. Material properties are ranked according to the resulting strain energy found in the blade model elements, when constructed entirely using a single material property. The strain energy, $\mathrm{U}\left[\mathrm{J} / \mathrm{m}^{2}\right]$, stored in a solid due to deformation under load is given by equation (1):

$$
U=\frac{1}{2} k \times \delta^{2},
$$

where $k[\mathrm{~N} / \mathrm{m}]$ is the material stiffness and $\delta[\mathrm{m}]$ is the displacement. The complete formulation of strain energy for composite materials can be found at [10]. Strain energy was chosen as the ranking criterion as opposed to blade stiffness, due to the multidirectional characteristic of the fibres in the composite materials present in wind turbine blades. The energy formulation of the laminate theory offers equivalent values of $E_{x}, E_{y}$ and $E_{z}$ for composite materials [10] with their magnitudes varying significantly depending on the type and lay of the composite material. Strain energy is a single scaler quantity that accounts for the energy stored in a solid due to the deformation of the solid in all directions, and therefore it is the more suitable material ranking criterion. Correctly ranking the material properties is a crucial step of setting up BELO, as the algorithm will place the stiffer materials in the areas of the model experiencing higher strain energy and the least stiff materials in areas of low strain energy. The next step of setting up the BELO algorithm consists of a heuristic process to determine the range of strain energy values experienced by the wind turbine blade. The strain energy range can be determined by observing the minimum and maximum strain energy values found in the blade model entirely constructed with a single material property. When recording the maximum and minimum strain energy values, it is important to observe and disregard unusual concentrations of strain energy in the model, as these are likely to be caused by singularities (for example, step changes in plate thickness due to changes in the number of glass layers). As in any other heuristic process, several iterations are required before deciding on the range of strain energy values to be used in the optimisation 
procedure. With the maximum and minimum strain energy values set, the strain energy range can be divided into intervals. The number of intervals must match the number of available materials properties, as every finite element in the blade model must always be assigned a material property. The stiffer material property is associated with the strain energy interval of highest value, the second stiffest material property is associated with the stain energy interval of second highest value and so on until all available material properties have been associated with one of the strain energy intervals. During the execution of the optimisation procedure, the finite elements in the blade model will be assigned with the material property corresponding to the strain energy interval in which they fit in. The magnitude of the strain energy intervals $g$, is defined by:

$$
g=\frac{U_{\max }-U_{\min }}{p-2},
$$

where $U_{\max }$ and $U_{\min }$ are the maximum and minimum strain energy values found in the blade model and $p$ is the number of available material properties.

If $i$ is the total number of finite elements in the blade model being optimised and $U_{i}$ is the strain energy in an element of the model, during the execution of the optimisation procedure, the material property should be assign to the element according to the following:

If $U_{i}<g \rightarrow$ assign weakest material property to the element.

If $g \leq U_{i}<2 g \rightarrow$ assign second weakest material property to the element.

If $U_{i} \geq g \times(p-1) \rightarrow$ assign stiffer material property to the element.

\subsection{Termination criterion}

In the original BESO method, the algorithm terminates when the prescribed volume for the structure is reached [6]. In the BELO method, there are no changes to the structure's volume and therefore a new termination criterion must be introduced. BELO algorithms will terminate if there is no significant change to the mean strain energy of the blade model, if a steady state is reached. The percentage of change $c$, of a successive number of iterations $j$, in the mean strain energy $U_{\text {blade, of }}$ the blade model are used to define the termination criterion, according to (3):

$$
c=\frac{\left|U_{\text {blade }(j-1)}-U_{\text {blade }(j)}\right|}{U_{\text {blade }(j)}} \times 100<e,
$$

The tolerance $e$, is user defined and its value should take into account the magnitude of the mean strain energy values in the wind turbine blade.

\subsection{BELO procedure summary and flow chart}

- Step 1: Create a finite element model of the wind turbine blade.

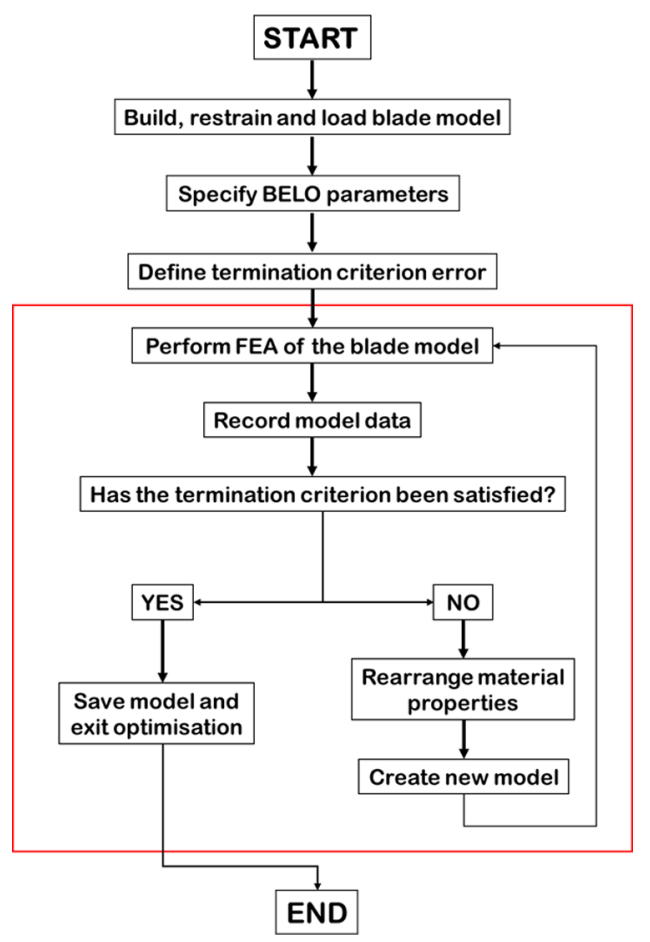

Fig. 1. BELO flow chart.

- Step 2: Load and restrain the model, it is recommended that the model be loaded with maximum operational load of the wind turbine blade.

- Step 3: Rank available composite material properties.

- Step 4: Specify the strain energy range, intervals and material properties associated with the strain energy intervals.

- Step 5: Specify parameters $e$ and $n$.

- Step 6: Carry out non-linear FEA of the initial blade model. A non-linear analysis is required to include the effects of centrifugal stiffening from blade rotation.

- Step 7: Input FEA results into BELO algorithm.

- Step 8: Rearrange material properties within the blade model according to FEA results.

- Step 9: Calculate mean strain energy of the blade model.

- Step 10: If termination criterion is meet, exit the optimisation loop.

- Step 11: If termination criterion is not meet return to step 6 and carry out FEA of the current blade model.

Figure 1 shows the optimisation method flow chart.

\section{Results}

The Aerogenesis $5 \mathrm{~kW}$ wind turbine is a two-bladed horizontal axis wind turbine that can be found at the University of Newcastle campus. The small wind turbine has been extensively instrumented and is primarily operated for experimental research work. Each of its current blades have a mass of just over $6 \mathrm{~kg}$ and are $2.5 \mathrm{~m}$ long, with the distance between blade tip and tower being approximately $600 \mathrm{~mm}$. The blades are composite-constructed, with a fibreglass outer shell and a foam core. Figure 2 a shows the cross section of the physical blade and Figure $2 \mathrm{~b}$ the finite element model 

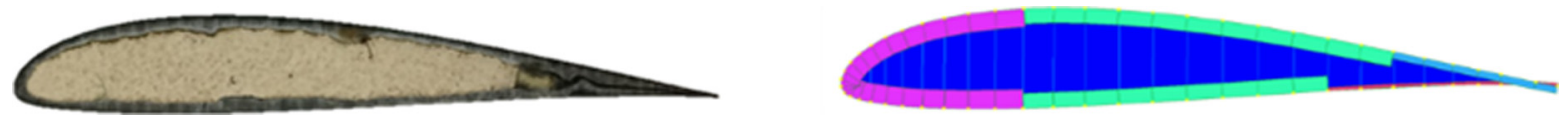

Fig. 2. (a) Physical blade cross-section and (b) blade model cross-section.

Fig. 3. Blade model.

Table 1. Material properties engineering data and ranking.

\begin{tabular}{llllll}
\hline Property rank number & $E_{x}(\mathrm{GPa})$ & $E_{y}(\mathrm{GPa})$ & $G_{x y}(\mathrm{GPa})$ & Density $\left(\mathrm{kg} / \mathrm{m}^{3}\right)$ & Thickness $(\mathrm{mm})$ \\
\hline 1 & 24.63 & 19.06 & 7.28 & 1789.03 & 6.2 \\
2 & 43.50 & 9.311 & 6.93 & 1774.18 & 3.95 \\
3 & 46.65 & 8.162 & 6.78 & 1796.57 & 3.5 \\
4 & 25.18 & 15.77 & 7.06 & 1756.36 & 4.4 \\
5 & 39.77 & 10.63 & 6.91 & 1752.44 & 3.07 \\
6 & 42.29 & 8.921 & 6.70 & 1778.63 & 2.62 \\
7 & 29.51 & 11.81 & 6.74 & 1717.48 & 2.26 \\
8 & 36.95 & 11.17 & 6.33 & 1742.53 & 1.74 \\
9 & 34.06 & 10.92 & 6.37 & 1746.69 & 1.81 \\
10 & 36.43 & 7.27 & 5.76 & 1792.25 & 1.29 \\
11 & 15.99 & 15.99 & 5.27 & 1632.56 & 0.09 \\
\hline
\end{tabular}

of the blade. Figure 3 shows the finite element model of the complete Aerogenesis blades with the different colours indicating a different fibreglass layup.

The load used in this optimisation of the Aerogenesis $5 \mathrm{~kW}$ blades represents the wind turbine's maximum operational load corresponding to a wind speed of $10.5 \mathrm{~m} / \mathrm{s}$, tip speed ratio of 8 (defined as the ratio of the tangential velocity of the blade tip divided by the upstream wind velocity) with the effects of centrifugal loading also included. At this point, it is important to mention that only the materials found on the outer shell of the blade will be optimised as the foam core of the blade has very low density and therefore its mass has little impact on the total blade mass. Fatigue data acquired prior from this study has the maximum fibre strain of approximately 2200 micro strain for a fatigue life of $10^{6}$ cycles [11]. Table 1 summaries the engineering data of the materials found in the Aerogenesis blade outer shell and their ranking according to strain energy. Figure 4 shows how the change in material property (i.e. change in stiffness) affects the resulting strain energy in the finite elements of the blade model. Element 2638 is located on the pressure surface of the blade in a region of high aerodynamic pressure.

As mentioned before, BELO procedures require an initial and accurate finite element model of the wind turbine blade. At the beginning of the optimisation procedure, all elements in the blade model must have a material property assign to it, in order to perform FEA and begin the procedure. The optimisation of the Aerogenesis $5 \mathrm{~kW}$ blade using BELO has proven effective regardless of the material property chosen to construct the initial blade model. All available material properties in the Aerogenesis blade were tested as the starting property of the optimisation procedure and all tests arrived at equivalent model solutions. Figure 5 shows stages of the optimisation procedure from two different starting material properties. Table 2 has a summary of the equivalent optimum layouts characteristics and FEA results of all optimum models of the Aerogenesis $5 \mathrm{~kW}$ blade, when subjected to the maximum operational load of the wind turbine.

To validate the Aerogenesis $5 \mathrm{~kW}$ blade model for the optimisation procedure, experimental data acquired by [11] prior to this study was compared to FEA results. The current blades of the Aerogenesis wind turbine are fitted with strain gauges along its radial length. The experiment measured deflection and strain recorded by the strain gauges when the blade was subjected to a point load applied close to its tip, vertically and onto the blade's pressure surface. During the experiment, three different load magnitudes $(3.139 \mathrm{~kg}, 5.234 \mathrm{~kg}$ and $7.226 \mathrm{~kg})$ were used and these conditions were simulated in the FEA of the blade. To further investigate the blade optimised layout characteristics, the optimised model was subject to the same point loads used to calibrate the initial model. Figure 6 shows the experimental results and FEA results of the physical blade and optimised blade model. These results show good correlation between experimental and FEA of the current blade layout, as well as good agreement with the expected results from FEA of the optimised blade model. 


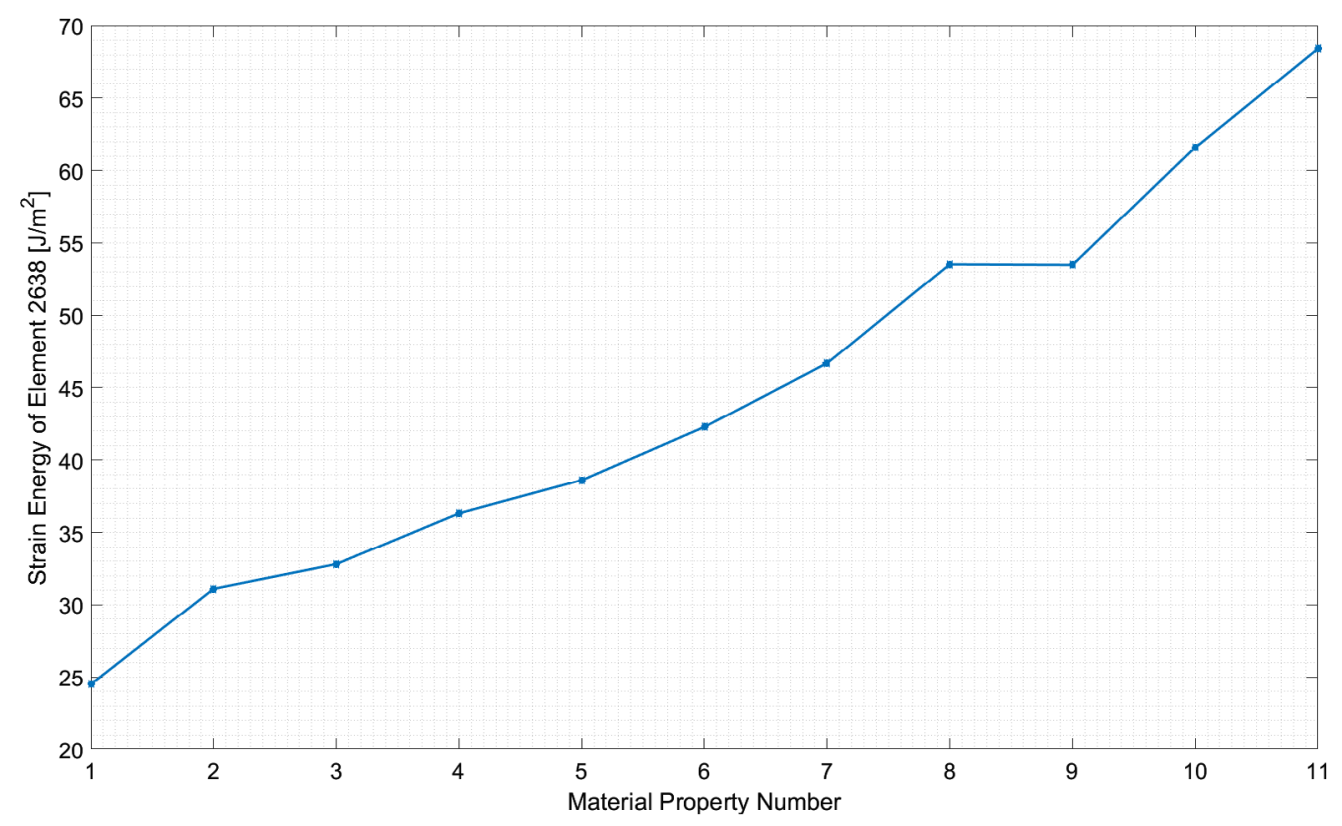

Fig. 4. Resulting strain energy in element number 2638.

First Iteration

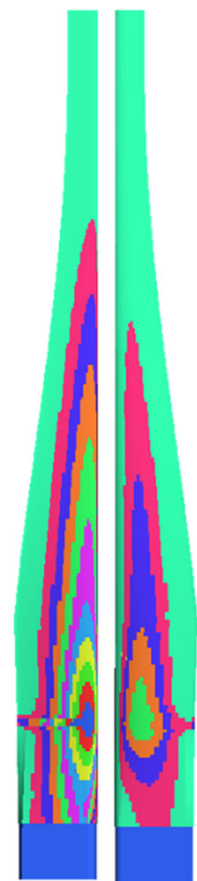

Starting Property: 2

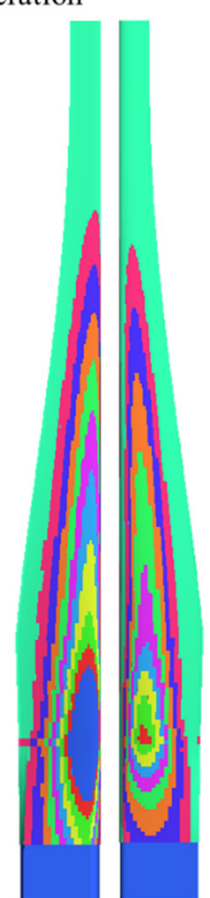

Starting Property: 8
Tenth Iteration

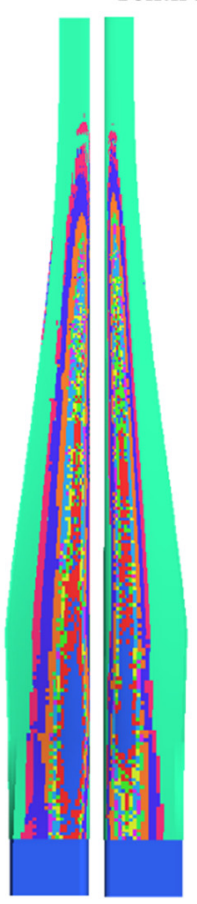

Starting Property: 2

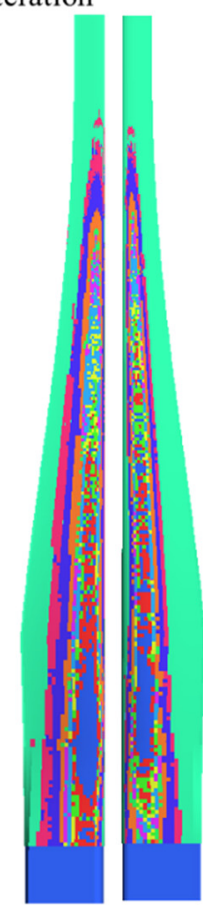

Starting Property: 8

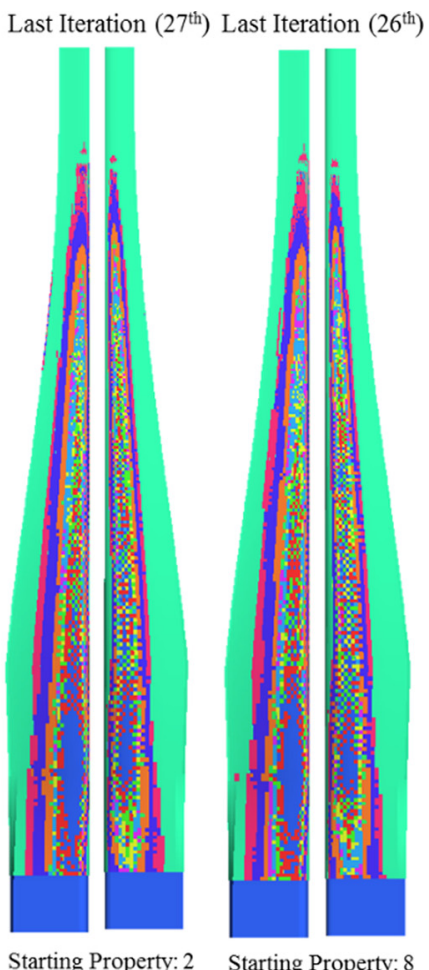

Fig. 5. Three stages of the Aerogenesis $5 \mathrm{~kW}$ blade optimisation, with pressure surface on the left of each pair and suction surface on the right.

\section{Discussion}

This paper presented a method to optimise the materials layout of wind turbine blades. The optimisation objective is set to reduce blade mass without exceeding maximum operational displacement and without reducing the blade's lifespan.
The optimisation results in Table 2 show a blade mass reduction of $14.5 \%$, from $6.01 \mathrm{~kg}$ of the current blade to $5.15 \mathrm{~kg}$ for the optimised blade and blade inertia reduction of approximate $19 \%$, from $5.79 \mathrm{~kg} \mathrm{~m}^{2}$ to $4.7 \mathrm{~kg} \mathrm{~m}^{2}$ in the axis of rotation. Also in Table 2, the maximum tip deflection and strain experienced by the optimised blade when subjected to maximum operational load are presented. The maximum tip deflection observed in the optimised blade models is $170 \mathrm{~mm}$ 
Table 2. Equivalent optimum models data.

\begin{tabular}{llllc}
\hline $\begin{array}{l}\text { Starting material } \\
\text { property number }\end{array}$ & $\begin{array}{l}\text { Model mass } \\
{[\mathrm{kg}]}\end{array}$ & $\begin{array}{l}\text { Model inertia } \\
{\left[\mathrm{kg} \mathrm{m}^{2}\right]}\end{array}$ & $\begin{array}{l}\text { Maximum tip } \\
\text { deflection [mm] }\end{array}$ & $\begin{array}{c}{ }^{*} \text { Strain on the 1st structural } \\
\text { ply [micro strain] }\end{array}$ \\
\hline 1 & 5.14 & 4.70 & 169.98 & 957.46 \\
2 & 5.14 & 4.71 & 169.89 & 1042.11 \\
3 & 5.14 & 4.71 & 169.70 & 1029.95 \\
4 & 5.14 & 4.70 & 169.87 & 977.44 \\
5 & 5.15 & 4.71 & 170.08 & 916.62 \\
6 & 5.15 & 4.71 & 169.72 & 1037.43 \\
7 & 5.14 & 4.70 & 169.56 & 997.06 \\
8 & 5.14 & 4.71 & 169.44 & 1007.44 \\
9 & 5.14 & 4.71 & 169.80 & 1008.93 \\
10 & 5.14 & 4.71 & 169.63 & 1008.52 \\
11 & 5.14 & 4.71 & 169.99 & 995.79 \\
\hline
\end{tabular}
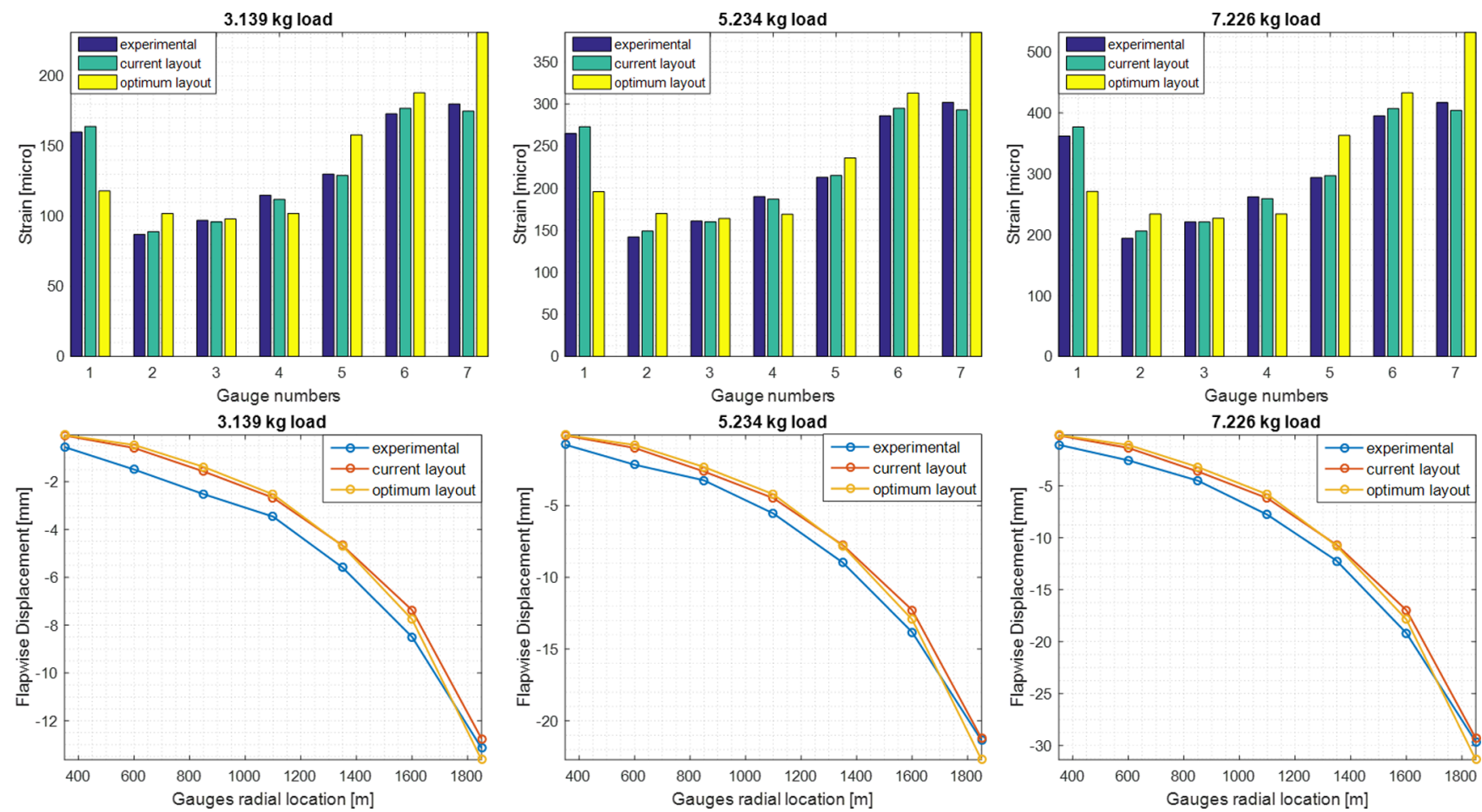

Fig. 6. Experimental data and FEA results from current blade layout and optimised layout.

and is well inside the $600 \mathrm{~mm}$ clearance between blade tip and tower. On close inspection of the optimised models, it was observed that the maximum strain energy values resulting from FEA did not correctly represent the maximum strain energy experienced by the blade, instead they are unusually high due to strain energy concentrations. As previously mentioned, concentration of strain energy can occur in FEA due to geometrical discontinuity between elements constructed with different material properties. Although some of the maximum strain values found in the optimised models exceed the proposed strain limit, the models are still considered to be acceptable solutions to the optimisation problem, as explained below Table 2. The strain in Figure 6 results shows a consistent trend, where the optimum layout experience lower strain than the current blade at the region closer to root. In addition, the optimum lay-up experiences much higher strain than the current blade at the blade region near the tip. Displacement results show very little discrepancy between optimum and current layout of materials. Analysis of the results presented in Figure 4 further validates the optimisation process, as mass reduction of almost a kilogram significantly changes the strain experienced by the blade material (particular close to blade root and tip) with very little effect on blade deflection.

The BELO method proved its effectiveness in the optimisation of the Aerogenesis blade. However, BELO is in its fundamental stages and it can be developed to be a more robust technique. Further refinement of the process 
that determines the strain energy range and its intervals is required. In its current version, BELO divides the strain energy range in intervals of the same magnitude. Preliminary examination of pressure distribution around large wind turbine blades suggests that it may be necessary to have intervals of different magnitudes. The technique can also be improved so the algorithm can automatically discard inefficient material properties during the execution of the procedure. A material property would be considered inefficient if it has not been sufficiently used during the optimisation. Another improvement relating to automation of the process is automating the acquisition of the resulting strain values in the blade model to improve accuracy of the observed strain quantity. Inclusion of buckling analysis to the optimisation procedure is also desirable. Lastly and perhaps the most challenging of improvements for BELO would be to have the optimisation procedure terminate with a manufacturable material layout for a wind turbine. Similar to BESO algorithms used to optimise composite constructed structures, the optimum layout of the structure it is not always manufacturable [9].

\section{References}

1. IEC 6140 0-2:2013 Standard for Small Wind Turbines
2. P. Brøndsted, R.P.L. Nijssen, Advances in Wind Turbine Blade Design and Materials (Woodhead Publishing Series in Energy, Cambridge, UK, 2013), Vol. 47

3. S.V.R. Wilson, P.D. Clausen, D.H. Wood, Gyroscopic moments on small wind turbine blades at high yaw rates. Aust. J. Mech. Eng. 5, 1 (2008)

4. M.O.L. Hansen, Aerodynamics of Wind Turbines, 2nd edn. (Earthscan, London, 2008)

5. O.M. Querin, G.P. Steven, Y.M. Xie, Evolutionary structural optimisation (ESO) using a bidirectional algorithm. Eng. Comput. 15, 1031 (1998)

6. L. Xia, Q. Xia, X. Huang, Y.M. Xie, Bi-directional evolutionary structural optimization on advanced structures and materials: a comprehensive review. Arch. Comput. Methods Eng. 1 (2016)

7. X. Huang, Y.M. Xie, M.C. Burry, A new algorithm for bidirectional evolutionary structural optimization. JSME Int. J. Ser. C 49, 1091 (2006)

8. D.R. Bradney, PhD Thesis, The University of Newcastle, Newcastle, Australia, 2017

9. X.F. Sun, J. Yang, Y.M. Xie, X. Huang, Z.H. Zuo, Topology optimization of composite structure using bi-directional evolutionary structural optimization method. Proc. Eng. 14, 2980 (2011)

10. J.M. Berthelot, Composite Materials: Mechanical Behavior and Structural Analysis (Springer Science \& Business Media, Berlin, Germany, 2012)

11. S.P. Evans, PhD Thesis, The University of Newcastle, Newcastle, Australia, 2017

Cite this article as: Mariana S.P. Costa, Samuel P. Evans, David R. Bradney, Philip D. Clausen, A method to optimise the materials layout of small wind turbine blades, Renew. Energy Environ. Sustain. 2, 19 (2017) 